\title{
Effects of exercise intensity on copy number and mutations of mitochondrial DNA in gastrocnemus muscles in mice
}

\author{
XIA CAO $^{1}$, ZHI-WEI ZHAO ${ }^{1}$, HONG-YING ZHOU ${ }^{1},{\text { GUO-QING } \text { CHEN }^{2} \text { and HUI-JUN YANG }}^{1}$ \\ ${ }^{1}$ Department of Human Anatomy, West China School of Preclinical and Forensic Medical Institute, \\ Sichuan University, Chengdu, Sichuan 61004; ${ }^{2}$ Department of Sports Anatomy, Wuhan \\ Institute of Physical Education, Wuhan, Hubei 430079, P.R. China
}

Received January 12, 2012; Accepted May 2, 2012

DOI: $10.3892 / \mathrm{mmr} .2012 .913$

\begin{abstract}
The present study aimed to investigate the effects of exercise intensity on mitochondrial DNA (mtDNA) alterations of copy numbers and mutations in the gastrocnemii of mice. A total of 50 male mice were randomly divided into 5 groups; control group (K) and groups A-D, which underwent 10,30,60 and 90 min of swimming per day, respectively. Samples were obtained after 20 weeks of exercise. Total DNA was collected to analyze mutations in the mtDNA displacement loop (D-loop) regions. mtDNA content was quantified by real-time PCR. Point mutations, monobase insertions and deletions were observed in the mtDNA D-loop regions in the skeletal muscles of the mice. A deletion of 16,232 bp was found in certain groups. The mutation base of the control group was higher than that of the exercise groups. The exercise groups demonstrated significantly altered copy numbers; the $30 \mathrm{~min}$ exercise group had the highest copy number. These results suggest that moderate exercise intensity reduces mutations in the mtDNA D-loop regions, and enhances the copy number of mtDNA in the gastrocnemus muscles of mice.
\end{abstract}

\section{Introduction}

Mitochondria are unique organelles as they contain their own DNA. The mitochondrial genome is a double-stranded circular DNA which is composed of coding and non-coding regions $(1,2)$. Mitochondria are important in energy metabolism, oxidative stress and cell apoptosis. Mitochondrial replication and synthesis are regulated by multiple factors. Replication of mitochondrial DNA (mtDNA) may be important in the maintenance of mtDNA copy number. The displacement loop (D-loop) region is the major control site for

Correspondence to: Professor Hui-Jun Yang, Department of Human Anatomy, West China School of Preclinical and Forensic Medical Institute, No.17 Renmin South Road, Chengdu, Sichuan 610041, P.R. China

E-mail: he500209@163.com

Key words: exercise, mitochondrial DNA, mutation, copy number, D-loop region
mtDNA replication and transcription (3). The D-loop region is increasingly susceptible to oxidative damage and electrophilic attack $(4,5)$, thus it is more prone to mutation. It has been acknowledged that exercise causes an increase in the skeletal muscle mitochondrial enzyme content and activity (6). Perturbations in mitochondrial content and function have been linked to a wide variety of diseases in multiple tissues, and exercise may serve as a potent approach by which to prevent and treat these pathologies (5). However, no study has been conducted to evaluate the association effects of exercise on mtDNA content and mutations. In this study, we investigated the effects of exercise intensity on mtDNA alterations of copy numbers and mutations in the gastrocnemii of mice.

\section{Materials and methods}

Animals and exercise protocol. Fifty male C57BL/6 mice, with an average weight of $15 \pm 2 \mathrm{~g}$, were used in this experiment. All animal use and protocols were approved by the Sichuan University, Sichuan, China. Animals were 4 weeks old at the beginning of the study. All animals were maintained at room temperature on a $12 \mathrm{~h}$ light-dark cycle, with free access to standard chow and water. The animals were randomly divided into five groups $(n=10)$; group $A, 10$ min of swimming per day; group B, $30 \mathrm{~min}$; group C, $60 \mathrm{~min}$; group D, 90 min and control group (group K). All mice in the exercise groups swam five times per week for 20 weeks. All animals were sacrificed $48 \mathrm{~h}$ after the last swimming session in order to examine the effect of total exercise, but not the effects resulting from the last swim. The gastrocnemii were carefully dissected, frozen in liquid nitrogen and stored at $-80^{\circ} \mathrm{C}$ until further analysis.

Detection of mtDNA content. Total DNA was isolated using the TIANamp Genomic DNA kit (DP110413; TianGen Biotechnology, Beijing, China). The mtDNA content was measured using real-time PCR. The primers used are listed in Table I. A standard curve was constructed using known concentration genomic DNA as a standard. QPCR was performed using an ABI 7900HT Fast Real-Time PCR System (Applied Biosystems, Carlsbad, CA, USA). The PCR conditions were: $2 \mathrm{~min}$ at $94^{\circ} \mathrm{C}$, followed by 40 cycles of $15 \mathrm{sec}$ at $94^{\circ} \mathrm{C}, 15 \mathrm{sec}$ at $58^{\circ} \mathrm{C}$ and $20 \mathrm{sec}$ at $68^{\circ} \mathrm{C}$; and 
Table I. Primers used for real-time PCR.

\begin{tabular}{llc}
\hline Fragment & \multicolumn{1}{c}{ Primer sequences (5'-3') } & Product size (bp) \\
\hline D-loop region & F: CCAACTAGCCTCCATCTCATACTTC & 1185 \\
& R: GGACCAAACCTTTCTCTTTATGGGA & 112 \\
mtDNA & F: CACTCCTCGTCCCCATTCTA & \\
& R: ATGCCGTATGGACCAACAAT &
\end{tabular}

D-loop, displacement loop; mtDNA, mitochondrial DNA; F, forward; R, reverse.

Table II. Mutations in the mtDNA D-loop regions in the gastrocnemus of mice.

\begin{tabular}{|c|c|c|c|c|c|c|}
\hline \multirow[b]{2}{*}{ Nucleotide site } & \multirow{2}{*}{$\begin{array}{l}\text { AY } 172335 \text { sequence } \\
\text { in GeneBank }\end{array}$} & \multirow{2}{*}{$\frac{\text { Control group }}{\mathrm{K}}$} & \multicolumn{4}{|c|}{ Exercise group } \\
\hline & & & A & $\mathrm{B}$ & $\mathrm{C}$ & $\mathrm{D}$ \\
\hline 15560 & $\mathrm{C}$ & Ins $\mathrm{C}$ & & & & \\
\hline 16032 & $\mathrm{G}$ & - & & & & \\
\hline 16232 & A & - & & & - & - \\
\hline 16236 & $\mathrm{~T}$ & $\mathrm{~T}$ & & & - & \\
\hline 16293 & $\mathrm{~T}$ & $\mathrm{~T}$ & & $\mathrm{C}$ & & \\
\hline
\end{tabular}

mtDNA, mitochondrial DNA; D-loop, displacement loop. A, adenine; G, guanine; C, cytosine; T, thymine; Ins, base insertion; -, base deletion.

a melting curve was constructed. The mtDNA content was measured using the standard curve.

Detection of mtDNA mutations. PCR was performed for the whole length of the mtDNA D-loop region, which was amplified using a 2X Taq PCR master mix (KT201-02; TianGen) and the primers listed in Table I. The PCR conditions were as follows: pre-denaturation for $4 \mathrm{~min}$ at $95^{\circ} \mathrm{C}, 30$ cycles of $45 \mathrm{sec}$ at $95^{\circ} \mathrm{C}, 55 \mathrm{sec}$ at $58^{\circ} \mathrm{C}$ and $60 \mathrm{~min}$ at $72^{\circ} \mathrm{C}$, with a final extension of $10 \mathrm{~min}$ at $72^{\circ} \mathrm{C}$. PCR products for TA cloning, the positive bacilli, were sequenced using delivery sequencing.

Statistical analysis. All data are expressed as the mean \pm standard error of the mean (SEM). Statistical analysis was performed using the Wilcoxon signed-rank test and Fisher's exact probability test with SPSS 13.0. $\mathrm{P}<0.01$ was considered to indicate a statistically significant difference.

\section{Results}

The mtDNA D-loop regions were successfully amplified (Fig. 1). Homology analysis of the sequencing results was conducted at http://blast.ncbi.nlm.nih.gov. The results detected mutations, monobase insertions and deletions in the control group and groups C and D which underwent 60 and 90 min of exercise, respectively. There was no mutation detected in group A which underwent 10 min of exercise. In group B which underwent 30 min of exercise only one point mutation was identified (Table II).

The melting curve of the real-time PCR products demonstrated a single peak, indicating the high specificity of the

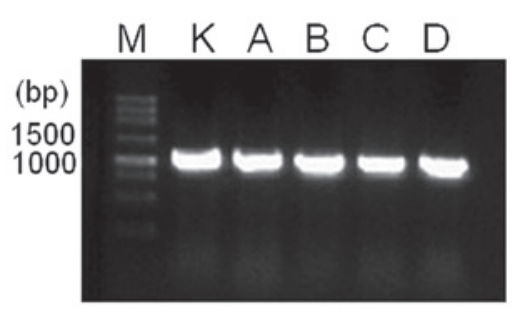

Figure 1. Gel electrophoresis of the mtDNA D-loop region in the gastrocnemii of mice. M, marker; K, control group; A, group A; B, group B; C, group C; D, group D. mtDNA, mitochondrial DNA; D-loop, displacement loop.

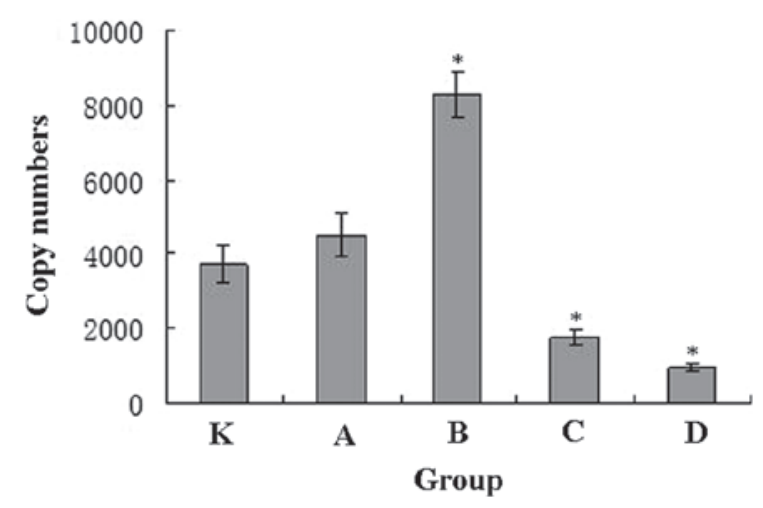

Figure 2. mtDNA contents in the gastrocnemii of mice (copies/ng). ${ }^{*} \mathrm{P}<0.01$ vs. K group. mtDNA, mitochondrial DNA; K, control group.

amplification. The standard curve correlation coefficient was 0.994 and the slope was -3.325 . Using the slope, the $\mathrm{Ct}$ values 
and concentrations of genomic DNA were used to calculate the mtDNA contents of each group (Fig. 2). The mtDNA content in groups $\mathrm{A}$ and $\mathrm{B}$, which underwent 10 and $30 \mathrm{~min}$ of exercise, was significantly increased, while the mtDNA content in groups $C$ and D, which underwent 60 and 90 min of exercise, was significantly decreased.

\section{Discussion}

Mitochondria are important in energy metabolism, oxidative stress and cell apoptosis. mtDNA is susceptible to damage by environmental carcinogens and endogenous reactive oxygen species (ROS) $(3,7)$, resulting in mtDNA mutations or base deletions. It has been confirmed that the mtDNA copy number and mtDNA mutations are related to disease and aging. Recent research has demonstrated that the mtDNA copy number is negatively correlated with tumor cell differentiation. The alteration in the mtDNA copy number may be an early event in oncogenesis. The mtDNA copy number decreases during aging. Mutated mtDNA may be preferentially amplified, and this increase might become clinically relevant after deconditioning (8). Considerable progress has been achieved towards the understanding of basic mitochondrial genetics, including the correlation between inherited mutations and disease phenotypes, and the identification of acquired mtDNA mutations during aging and cancer. The mtDNA D-loop is a non-coding sequence of the mitochondrial genome that is implicated in mtDNA replication and transcription. The $\mathrm{D}$-loop region is more susceptible to oxidative damage and electrophilic attack.

In this study, we sequenced the mtDNA D-loop region of mouse gastrocnemii and detected a point mutation, monobase insertion and deletion in the control group, the $60 \mathrm{~min}$ exercise group and the 90 min exercise group, respectively. Extensive exercise training can alter mutations in the mtDNA D-loop region of mouse gastrocnemus cells, suggesting that 10 and $30 \mathrm{~min}$ of exercise intensity is more appropriate than other exercise intensities for reducing mouse gastrocnemus mtDNA D-loop mutations, while high-intensity sports have a negative impact. Research has demonstrated that the accumulation of mtDNA mutations and apoptosis markers are closely related, therefore it has been speculated that the accumulation of mtDNA mutations caused by apoptosis, may be a core mechanism involved in aging and disease. In this study, 10 and $30 \mathrm{~min}$ per day of exercise training increased the relative mtDNA copy number, however, 60 and 90 min per day of exercise significantly reduced the mtDNA copy number; these results were in accordance with the results of the mtDNA D-loop region mutations. mtDNA mutations have the ability to cause injury to respiratory chain subunit protein synthesis, formation of a defective respiratory chain and reduction in the number of normal mitochondria (9). Accumulation of mtDNA mutations may cause apoptosis associated with reduced mitochondrial copy number. The direct impact of reduced mitochondrial copy number may reduce mitochondrial oxidative phosphorylation, resulting in decreased productivity, and promotion of ROS generation, resulting in decreased cell function and apoptosis and promotion in the occurrence of aging and disease. Moderate exercise can reduce damage caused by mtDNA mutations and increase the number of normal mitochondria. It may enhance the activity of mitochondria to prevent apoptosis, aging and diseases.

In conclusion, our study demonstrated that regular exercise could alter mtDNA mutations and mtDNA copy number. This may provide a new approach to enhance the function of mitochondrial oxidative phosphorylation for the prevention of disease and aging. Further investigations are required.

\section{Acknowledgements}

This study was supported by the Sichuan University Scientific Research Foundation for Young Teachers (2009SCU11145).

\section{References}

1. Shen L, Fang H, Chen T, et al: Evaluating mitochondrial DNA in cancer occurrence and development. Ann NY Acad Sci 1201: 26-33, 2010.

2. Bayona-Bafaluy MP, Acín-Pérez R, Mullikin JC, et al: Revisiting the mouse mitochondrial DNA sequence. Nucleic Acids Res 31: 5349-5355, 2003.

3. Suzuki M, Toyooka S, Miyajima K, et al: Alterations in the mitochondrial displacement loop in lung cancers. Clin Cancer Res 9: 5636-5641, 2003.

4. Mambo E, Gao X, Cohen Y, et al: Electrophile and oxidant damage of mitochondrial DNA leading to rapid evolution of homoplasmic mutations. Proc Natl Acad Sci USA 100: 1838-1843, 2003.

5. Park HW, Ahn Y, Jeong MH, et al: Chronic atrial fibrillation associated with somatic mitochondrial DNA mutations in human atrial tissue. J Clin Pathol 60: 948-950, 2007.

6. Little JP, Safdar A, Benton CR and Wright DC: Skeletal muscle and beyond: the role of exercise as a mediator of systemic mitochondrial biogenesis. Appl Physiol Nutr Metab 36: 598-607, 2011.

7. Lievre A, Chapusot C, Bouvier AM, et al: Clinical value of mitochondrial mutations in colorectal cancer. J Clin Oncol 23: 3517-3525, 2005.

8. Taylor RW and Turnbull DM: Mitochondrial DNA mutations in human disease. Nat Rev Genet 6: 389-402, 2005.

9. Richter CJ, Park BN and Ames BN: Normal oxidative damage to mitochondrial and nuclear DNA is extensive. Proc Natl Acad Sci USA 85: 6465-6467, 1988. 